\section{Reply to Makous ${ }^{1}$}

\author{
GERALD S. WASSERMAN, University of \\ Wisconsin, Madison, Wis. 53706
}

Makous (1970) makes several comments about our earlier paper (Wasserman \& Patton, 1969) that oversimplify the situation and require a reply. First, Makous is persuaded that Limulus reliably moves its tail downward in response to stimulation. This is not the case: In our work, the tail moved downward in response to light and upwards in response to shock. The upwards movements occurred for weak (0.1 mA) as well as strong $(50 \mathrm{~mA})$ shocks. We used a powerful shock simply because rapid habituation was observed for weak shocks. In Smith \& Baker's (1960) work, the tail always moved upward in response to both light and shock (Baker, personal communication). So the direction of tail movement is not invariant.

Second, and more importantly, there is the question of the probability of a tail movement, irrespective of its direction. Makous gives the impression that this "natural part of the animal's escape reaction" is a well-known and invariant consequence of stimulation. Had this been the case, it is very hard to understand why any of us would have tried to use tail movement as the response in a conditioning experiment. However, at the time of our report, the experimental evidence was that this response does not occur with any great frequency. In Smith and Baker's controls, the response frequency was about 30\%. In Makous's
(1969) second classical conditioning experiment, the response frequency was about $2.5 \%$. In our first set of sensitization and pseudoconditioning controls, the response frequency was also about $30 \%$. These first control experiments were completed prior to the appearance of our report in this journal, although they were not mentioned in that report. Had our control experiments turned out otherwise, we would have withdrawn our report prior to publication.

Subsequently, however, and in contradiction to both the earlier reports and our own experience, we have found that some, but not all, naive Limulus exhibit a tail movement in response to light alone, that this response can occur with frequencies well above $75 \%$, and that it persists without habituation for periods of several months. This behavior seems to be more profitably viewed as a fixed-action-pattern (FAP) that may or may not be expressed, depending on conditions that are not yet understood. In another paper (Wasserman \& Patton, in preparation), we have described these results in detail, as well as the use of the FAP to obtain a visual threshold. There is nothing in the experimental literature that might have suggested that such an obvious and persistent FAP involving tail movement could exist; however, analogous FAPs involving leg movements have been recently reported by Corning \& von Burg (1968), although they report rapid habituation of these leg-movement FAPs. Makous (1970), then, is certainly correct in questioning our report of successful conditioning, but for reasons other than those expressed. The essential point here is not that our work lacked the relevant controls, but that the relevant control procedures have given us different results with different groups of animals. The fact that some animals exhibit a high probability of tail movement to light alone renders it quite likely that the effect of pairing light with shock potentiates an otherwise unexpressed tail movement in animals that exhibit a low probability of tail movements to light alone. At this time, it is our belief that the existence of this FAP involving tail movement controverts all prior reports of associative conditioning in Limulus, and requires that we all change our ideas on this subject.

\section{REFERENCES}

CORNING, W. C., \& von BURG, R. Behavioral and neurophysiological investigations of Limulus polyphemus. In J. Salanki (Ed.), Neurobiology of invertebrates. New York: Plenum, 1968.

MAKOUS, W. L. Conditioning in the horseshoe crab. Psychonomic Science, 1969, 14, 4-6.

MAKOUS, W. L. Conditioning in Limulus: Comment on Wasserman and Patton. Psychonomic Science, 1970, 00, 000-000.

SMITH, J. C., \& BAKER, H. D. Conditioning in the horsehoe crab. Journal of Comparative and Phsyiological Psychology, 1960, 53, 279-281. WASSERMAN, G. S., \& PATTON, D. G. Avoidance conditioning in Limulus. Psychonomic Science, 1969, 15, 143. NOTES

1. This research was supported in part by the Wisconsin Alumni Research Foundation, in part by the Biomedical Sciences Support Grant administered by the University Graduate Research Committee, and in part by a grant from the National Science Foundation (GB-8581).

2. Wasserman, G. S., \& Patton, D. G. Visually guided behavior in Limulus: A fixed-action pattern elicited by light. In preparation. 